Check for updates

Cite this: Chem. Sci., 2019, 10, 4089

๑ All publication charges for this article have been paid for by the Royal Society of Chemistry

Received 2nd January 2019

Accepted 23rd February 2019

DOI: $10.1039 / \mathrm{c} 9 \mathrm{sc00020h}$

rsc.li/chemical-science

\section{AminoazobenzeneaAg modified meshes with large extent photo-response: towards reversible oil/water removal from oil/water mixtures $\uparrow$}

\begin{abstract}
Ruixiang Qu, ${ }^{a}$ Yanan Liu, ${ }^{a}$ Weifeng Zhang, ${ }^{a}$ Xiangyu Li, ${ }^{a}$ Lin Feng (D) *a and Lei Jiang (D) ${ }^{b}$
Photo-responsive materials with superwetting properties, especially in the azo-based class, have been used in water treatment because of their smart performance on wettability changes. However, their transformation extent in wettability has always troubled researchers. Here, we modified nano-Ag pine needles and aminoazobenzene (AABN) on polydopamine (PDA) pre-treated porous meshes, realizing a large-extent reversible photo-responsive wettability transformation from highly hydrophobic to highly hydrophilic. The contact angle is about $150.0^{\circ}$ after being exposed to visible light, and is about $10.0^{\circ}$ under $365 \mathrm{~nm}$ UV light. Accordingly, the modified mesh can achieve photo-responsive removal between oil and water from oil/water mixtures. This facile and universal approach based on trans-cis isomerization of AABN could be endowed to various commercial conductive meshes. Moreover, the modified meshes exhibit satisfactory removal efficiency, reusability and physical/chemical stability, which are more promising for practical applications such as fuel recycling, remote controlled oil/water separation and astronautical resource regeneration.
\end{abstract}

\section{Introduction}

Due to their outstanding potential in water treatment, microfluidics, self-cleaning and molecular transportation, superwetting materials have been widely studied in the past few decades. ${ }^{1-7}$ Traditional superwetting materials such as superhydrophilic/underwater superoleophobic materials and superhydrophobic/superoleophilic materials are not enough to meet the complex requirements of practical application now. ${ }^{8-13}$ Thus, researchers tend to focus on smart superwetting materials with thermal-responsive, electro-responsive and pH-responsive properties. ${ }^{14-18}$ Among all of the stimuliresponsive materials, photo-responsive materials were an emerging class with attractive superiorities, which have become a hot research topic in recent years, especially in the field of oil/water separation. ${ }^{19-21}$ The utilization of photoswitchable materials can effectively avoid contact with materials during the response process, which decreases the materials' contamination and increases the reusability of the materials. Besides, owing to their short response time as well as reversibility, photo-responsive materials can realize wettability transformation rapidly and repeatedly, which effectively

a'Department of Chemistry, Tsinghua University, Beijing 100084, P. R. China. E-mail: fl@mail.tsinghua.edu.cn

${ }^{b}$ Key Laboratory of Bio-inspired Smart Interface Sciences, Technical Institute of Physics and Chemistry, Chinese Academy of Sciences, Beijing, 100084, P. R. China

$\dagger$ Electronic supplementary information (ESI) available. See DOI: 10.1039/c9sc00020h improves their separation efficiency. Furthermore, the mild response condition during the photo-response promises a greener and more economical approach to practical application. However, it is difficult to fabricate photo-responsive superwetting materials up to now, and there are still some challenges that need to be overcome such as exorbitant reagent prices, complicated fabrication processes and limited direction for application. Therefore, it is of great significance to propose a facile and low-cost approach to fabricate photoresponsive superwetting materials and tap their application potential.

Azo compounds, with their excellent cis-trans isomerism properties, have become star molecules for fabricating photoresponsive surfaces. ${ }^{22-25}$ Most of the azo compounds can realize molecular structure changes under UV and visible light. And the intricate mechanism of azo isomerization has been extensively studied in the past few decades. Currently, rotation of the $\mathrm{N}=\mathrm{N}$ bond, the inversion of the $\mathrm{NNC}$ plane and the concerted-inversion of the NNC plane are the three most accepted mechanisms. ${ }^{26,27}$ In spite of the excellent photoresponsive properties of azo compounds, it is often hard to modify azo compounds on a given substrate because the large molecular size and the stable benzene rings make the chemical reaction difficult. Besides, due to the extent of transformation of the azo molecular structure being limited, the wettability of photo-responsive azo surfaces still varies within a small range now, which severely impedes their practical application. For example, Xie et al. fabricated an azo/ $\beta-C D$ modified nanochannel, which could realize WCA change 
between $16.5 \pm 1.8^{\circ}$ and $67.3 \pm 0.2^{\circ} .^{28}$ Petroffe et al. coated azo/ $\mathrm{TiO}_{2}$ onto the substrates and realized a WCA variation of about $100^{\circ} .^{29}$ Therefore, how to fabricate an azo modified photoresponsive material with a large transformation extent is still a challenge.

Herein, we modified nano-Ag pine needles and AABN on PDA pre-treated meshes, and realized a large-extent photoresponsive wettability transformation. The Ag/AABN modified meshes showed high hydrophobicity $\left(\mathrm{WCA} \approx 150.0^{\circ}\right.$ ) and could remove water under visible light, while showed high hydrophilicity $\left(\mathrm{WCA} \approx 10.0^{\circ}\right.$ ) and could remove oil from an oil/water mixture under $365 \mathrm{~nm}$ UV light. Moreover, the wettability transformation was reversible, and the meshes possessed satisfactory removal efficiency, reusability and physical/chemical stability. In order to break through the transformation extent limitation of photo-responsive azo materials, AABN with its small molecule group was utilized to reduce the steric hindrance, and the fabrication condition of fractal $\mathrm{Ag}$ nanostructures was controlled to maximize the surface roughness. ${ }^{30,31}$ Besides, the fabrication approach was simple and universal and could be used for different conductive meshes such as copper meshes, stainless steel meshes and nickel meshes. Compared to the previous excellent work about photo-responsive azo compounds, this work showed the largest transformation extent, and could be applied in reversible removal between oil and water from oil/ water mixtures (Table S1†). ${ }^{28,29,32,33}$

As illustrated in Scheme 1, the substrate was pre-treated with PDA to endow the surface with a high adhesive, thus making the coatings more stable. ${ }^{34-36}$ After a well-regulated electrodeposition process, the fractal $\mathrm{Ag}$ pine nano-needles were modified on the substrate to form an extremely rough structure. Then the AABN was uniformly coated onto the surface by a simple immersion process. The AABN was connected to the $\mathrm{Ag}$ pine needles by the coordinate bond between $-\mathrm{NH}_{2}$ and $\mathrm{Ag} .{ }^{37,38}$ Here, the Ag pine needles provided hydrophilic groups and high surface roughness, while the AABN provided hydrophobic groups and a photo-responsive property. In the initial state, the AABN was in its trans-form with its molecule stood up straight, thus the hydrophobic benzene ring of AABN was exposed. Combining the high surface roughness provided by the Ag pine needles and the hydrophobicity of the benzene ring, the modified mesh showed a high degree of hydrophobicity. After being irradiated with $365 \mathrm{~nm}$ UV light, the $\mathrm{N}=\mathrm{N}$ bond of AABN was broken and rotated, so the AABN turned into its cis-form with its molecule lying down. Thus the hydrophilic Ag pine nano-needles were exposed, making the modified mesh highly hydrophilic. After irradiation with visible light, the mesh could turn back to its initial highly hydrophobic state, indicating the excellent reversible wettability transformation property.

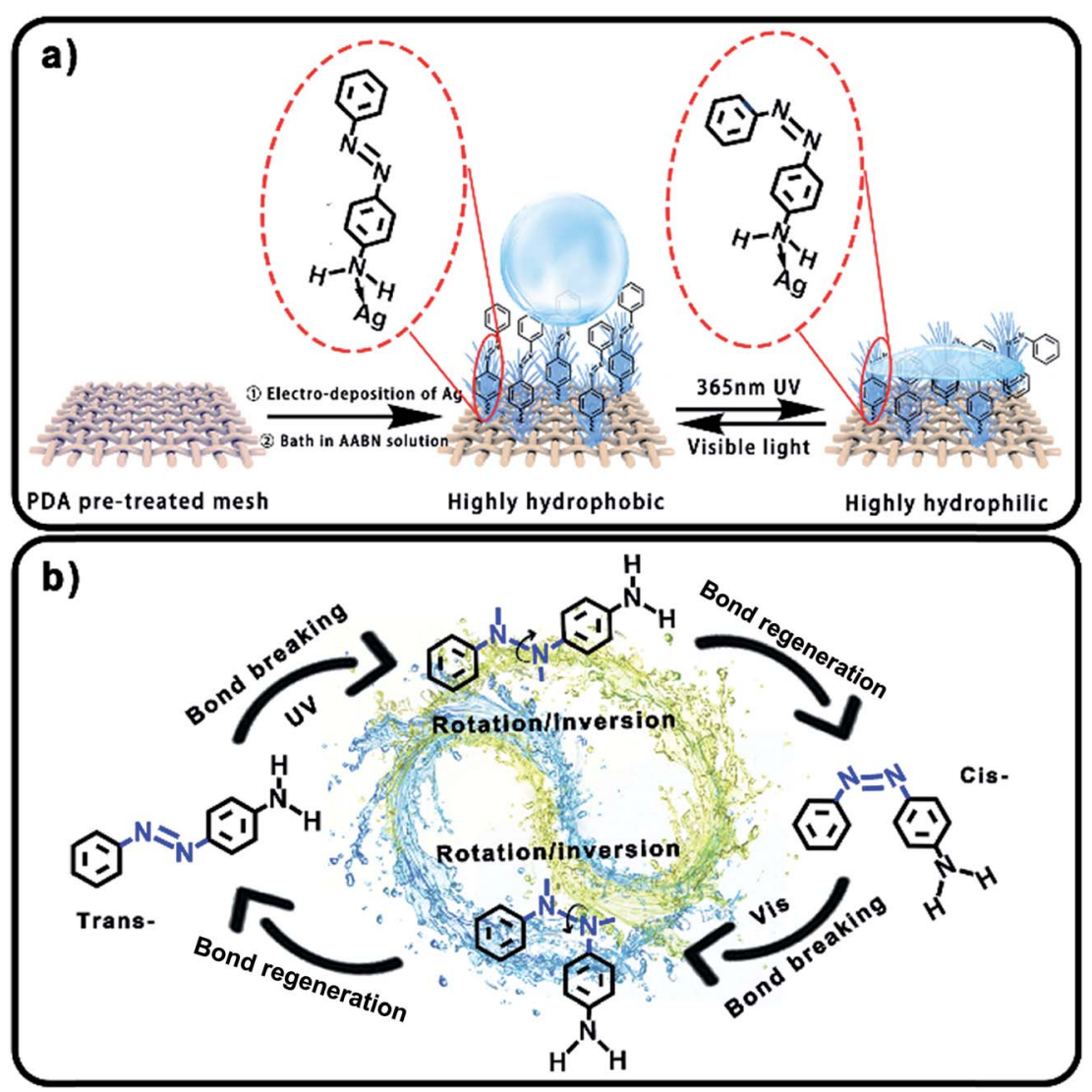

Scheme 1 (a) The modification process of conductive meshes and (b) the mechanism of photo-responsive wettability transformation. The wettability transformation property owed to the alternate exposure of the hydrophilic group (Ag) and hydrophobic group (aminoazobenzene). 


\section{Results and discussion}

\section{Surface morphology and chemical composition}

The modification of the commercial copper mesh was carried out first to verify the feasibility of the mentioned approach. Ag pine needles were uniformly coated on the copper mesh surface through an electrolytic deposition process, followed by AABN being steadily adhered to the Ag pine needles. Digital photos of the modified copper mesh in different reaction stages are shown in Fig. S1.† As seen, the yellowish original copper mesh turned gray after being modified with PDA and Ag pine needles, and the final mesh was clay coloured due to the orange AABN covering the gray Ag. The morphologies of the substrate before and after anchoring with AABN were observed using a Field Emission Scanning Electron Microscope (FESEM). Fig. 1a and b show typical images of the Ag pine needle-coated copper substrate with a rough surface at different magnifications. Fractal $\mathrm{Ag}$ pine needles with an average size of $10 \mu \mathrm{m}$ were densely grown on the substrate. The smallest unit of the fractal was $200 \mathrm{~nm}$. After modification with $\mathrm{AABN}$, the microstructure of the surface remained unchanged because the AABN coating was too thin to be observed using SEM (Fig. 1c), and further characterization was needed to prove the successful modification of AABN as follows. Fig. S2 $\uparrow$ shows the SEM image of the cross section of the modified copper mesh, indicating that the surface of the mesh was rough and the height of the Ag pine needles was $1-10 \mu \mathrm{m}$.

The elemental composition and surface structure of the substrate before and after modification were detected using Xray Photoelectron Spectroscopy (XPS), X-ray Diffraction (XRD) and Energy Dispersive X-ray Spectroscopy (EDX). Fig. 1d depicts the XPS spectra of the copper before and after anchoring with AABN. The peaks at $368.4 \mathrm{eV}, 284.9 \mathrm{eV}$, and $399.6 \mathrm{eV}$ were ascribed to Ag3d, C1s and N1s, respectively. ${ }^{39-41}$ A clear change in the elemental ratio after AABN coating was obvious, which was caused by the high content of $\mathrm{C}$ and $\mathrm{N}$ in $\mathrm{AABN}(73.0 \%$ and $21.3 \%$ ), as calculated from its monomer. Besides, the $\mathrm{Ag}$ signal peak reduced after AABN coating, which was due to the AABN molecules covering the Ag pine needles. Fig. S3† exhibits the narrow spectrum of the N1s orbit; the chemical shift of $\mathrm{N}$ indicated its valance change, which hinted at the formation of the coordination bond between $\mathrm{AABN}$ and the $\mathrm{Ag}$ pine needles. ${ }^{\mathbf{4 2}}$ Fig. 1e shows the XRD pattern of the copper mesh in different reaction stages. The diffraction peaks in the pattern could be indexed to silver (JCPDS-04-0783). ${ }^{43}$ The Ag peak appeared after the mesh was modified with Ag pine needles, and reduced after the $\mathrm{Ag}$ pine needles were covered by AABN. Fig. 1f shows the corresponding mapping images of the mesh. After anchoring with $\mathrm{AABN}$, the Ag signal remained strong while the $\mathrm{N}$ signal was obviously enhanced. Fig. S4† exhibits the EDX images of the modified copper mesh with and without AABN. The mass fraction of $\mathrm{Ag}$ decreased to $31.6 \%$ from $37.3 \%$ after $\mathrm{Ag}$ was covered with $\mathrm{AABN}$, and the fractions of $\mathrm{C}$ and $\mathrm{N}$ increased to varying degrees due to the presence of AABN. Besides, the SEM image of the $\mathrm{Ag}$ modified copper mesh without the pretreatment of PDA was also collected to confirm the necessity of the adhesive PDA. As shown in Fig. S5, $\dagger$ the surface roughness was low and the $\mathrm{Ag}$ pine needles could not evenly coat the mesh surface without PDA. Fig. S6 $†$ shows the UV spectra of the AABN solution before and after the reaction. An $11.3 \%$ deduction of the spectrum indicated that $2.20 \mathrm{mg}$ of AABN was coated on the copper mesh. In this way we could confirm the successful modification of the Ag pine needles and AABN.

\section{Photo-responsive wettability transformation}

Due to the sensitive photo-responsive property of the azo group in $\mathrm{AABN}$, the $\mathrm{Ag} / \mathrm{AABN}$ modified mesh showed a reversible wettability transformation property, i.e., it was highly
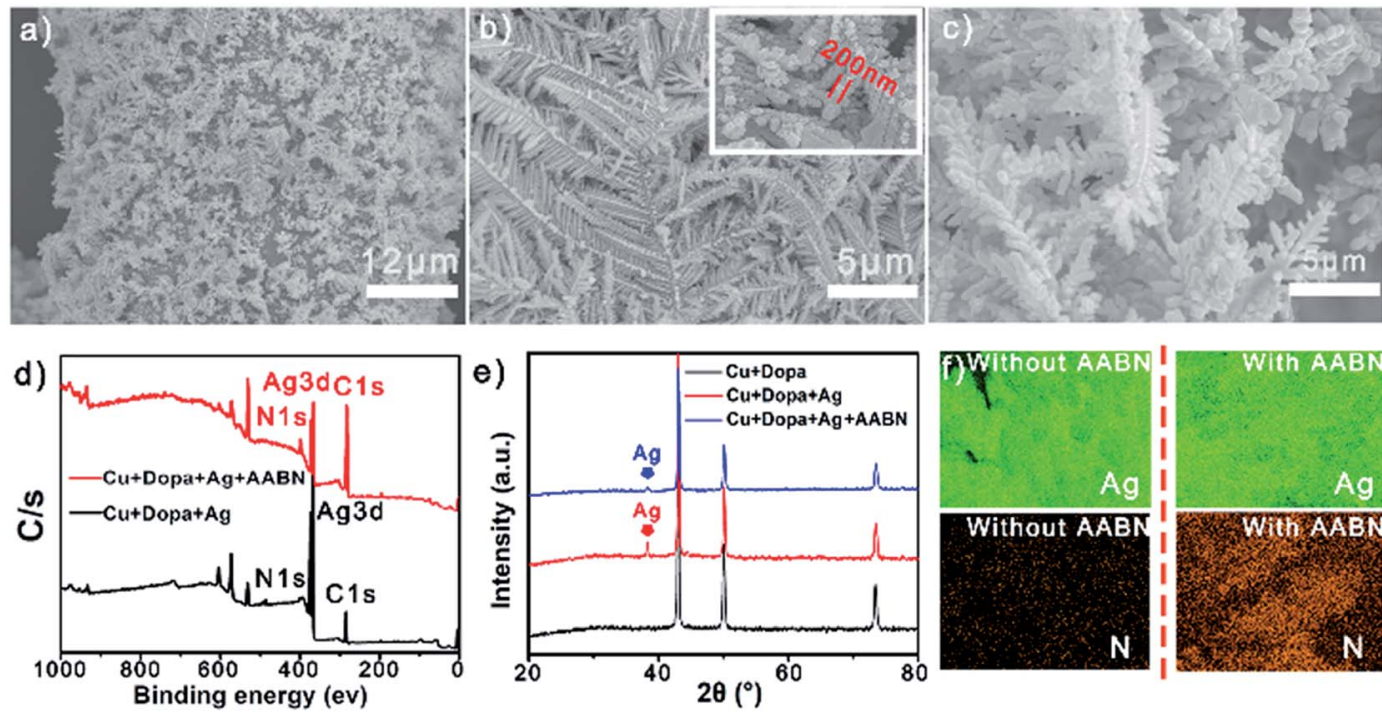

Fig. 1 The morphology and chemical composition: (a and b) SEM images of Ag pine needle modified copper mesh; (c) SEM image of AABN/Ag modified copper mesh; (d) XPS pattern of modified copper; (e) XRD pattern of modified copper in different reaction stages; (f) elemental mapping images of elements $\mathrm{Ag}$ and $\mathrm{N}$ on the modified copper mesh. 
hydrophilic under UV light while highly hydrophobic under visible light. The large extent wettability transition was governed by two factors: one was the utilization of small-molecule AABN reducing the steric hindrance, and the other was the fractal $\mathrm{Ag}$ pine needles enhancing the surface roughness. In order to obtain the optimal meshes, the Ag fabrication conditions were adjusted under $1.5 \mathrm{~V} / 3 \mathrm{~min}, 1.5 \mathrm{~V} / 6 \mathrm{~min}, 3 \mathrm{~V} / 3 \mathrm{~min}$ and $3 \mathrm{~V} / 6 \mathrm{~min}$, which showed average WCAs of $119.8^{\circ}, 152.8^{\circ}$, $127.0^{\circ}$ and $148.7^{\circ}$, respectively (Fig. 2a). The mesh fabricated under $1.5 \mathrm{~V} / 6 \mathrm{~min}$ showed the largest WCA in its initial state, which corresponds with the highest roughness SEM images shown in Fig. S7.† The semi-quantitative roughness of the meshes calculated using Young's equation is exhibited in Fig. S8. $\dagger$ The roughness of the mesh fabricated under $1.5 \mathrm{~V} /$ 6 min was nearly double those fabricated under $1.5 \mathrm{~V} / 3 \mathrm{~min}$ and $3 \mathrm{~V} / 3 \mathrm{~min}$, and a little higher than that fabricated under $3 \mathrm{~V} /$ $6 \mathrm{~min}$. It is considered that reducing the deposition time could not support the formation of $\mathrm{Ag}$ pine needles while increasing the deposition voltage would turn the $\mathrm{Ag}$ into clusters, which both make the mesh not rough enough. The more detailed relationship between $\mathrm{Ag}$ fabrication conditions and WCA/ surface roughness was investigated and is shown in Fig. S9, $\dagger$ in which we could find that the meshes fabricated under $1.5 \mathrm{~V} /$ 6-8 min all showed high WCA and surface roughness. Besides, the transformation extent of the mesh severely decreased without PDA (Fig. S10 $\dagger$ ). As a result, we could confirm that $1.5 \mathrm{~V} /$ 6 min was the optimum and selected the mesh prepared under this condition to further investigate.

Fig. $2 \mathrm{~b}$ and $\mathrm{S} 11 \dagger$ show the reversible wettability transformation of the modified $\mathrm{Ag} / \mathrm{AABN}$ copper mesh. The newly prepared mesh was highly hydrophobic/superoleophilic because the hydrophobic trans-AABN covered the hydrophilic
Ag. The WCA and oil contact angles (OCA) of the initial mesh were $147.2^{\circ}$ and $0^{\circ}$, respectively. After being exposed to UV light for 20 minutes, the mesh turned highly hydrophilic/underwater highly oleophobic so that the water drop could spread out on the mesh $\left(\mathrm{WCA}=12.1^{\circ}\right.$, underwater $\left.\mathrm{OCA}=138.0^{\circ}\right)$. Then the highly hydrophilic mesh was exposed to visible light for 20 minutes to turn back to highly hydrophobic $\left(\mathrm{WCA}=149.6^{\circ}\right.$, $\mathrm{OCA}=0^{\circ}$ ). The irradiation process is shown in Fig. S12 $\dagger$ with digital photos. Besides, the wettability transformation process was reversible under alternate UV/Vis light. Fig. 2c shows five successive wettability transformation cycles of the modified copper mesh. The mesh remained Vis/highly hydrophobic and UV/highly hydrophilic after five cycles. Fig. S13† exhibits the contact angles of four different oils ( $n$-hexane, toluene, gasoline and diesel) under water of the modified copper mesh after UV irradiation; all of the contact angles were higher than $135.0^{\circ}$. To better embody the wetting behavior transformation property of the mesh, half of a modified copper mesh was kept away from light while another half of the mesh was exposed to UV light. The mesh would show different wetting behavior in its different parts (Fig. S14 $\dagger$ ). Besides, in order to confirm the necessity of AABN, AABN@Ag modified meshes fabricated under different AABN concentrations were collected and their water contact angles under visible light were measured. As shown in Fig. S15, $\dagger$ when the AABN concentration was $2 \mathrm{mM}$, the as-prepared mesh showed a $150^{\circ}$ contact angle under visible light. However, when the AABN concentration was lower than $2 \mathrm{mM}$, the contact angles under visible light were not high enough to meet the requirement for oil/water separation. Besides, the structural change of AABN upon exposure to different stimuli was proved using a UV/Vis spectrophotometer, as shown in Fig. S16. $\dagger$ The AABN was dissolved in $N, N$-dimethyl formamide (DMF) during
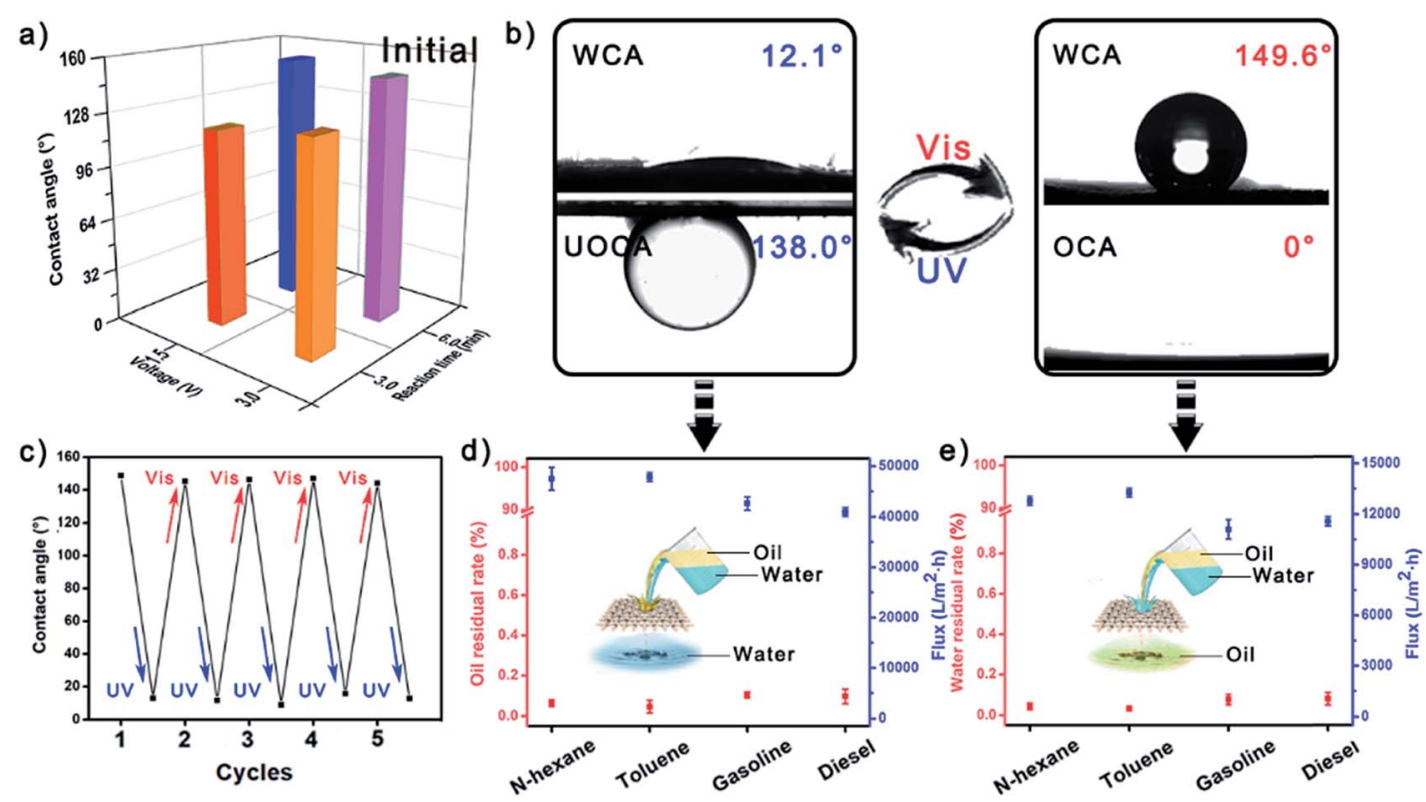

Fig. 2 The photo response performance: (a) the initial contact angles of the modified meshes fabricated under different conditions; (b) the WCA/ OCA of the modified copper mesh; (c) five successive wettability transformation cycles; (d) oil removal test after UV irradiation; (e) water removal test after visible light irradiation. 
the test. The absorption peak was at $401.02 \mathrm{~nm}$ when AABN was illuminated by visible light. And after the AABN was exposed to UV light for 20 minutes, a new absorption peak at $364.20 \mathrm{~nm}$ appeared while the peak at $401.02 \mathrm{~nm}$ reduced by $50 \%$, which indicated that the AABN turned from its trans-form to cis-form under UV light. As a result, we could demonstrate the reversible wettability transformation of the modified Ag/AABN copper mesh.

\section{Selective removal of oil or water}

Owing to the excellent photo-responsive wettability transformation property of the modified copper mesh, it was expected to realize selective oil/water removal under UV/Vis light. By simply pouring an oil/water mixture onto the mesh, either the oil or water phase could pass through the mesh while the other phase would be isolated and removed, depending on the wetting behavior of the mesh. After being exposed to UV light for 20 minutes, the modified mesh was highly hydrophilic and could remove oil from an oil/water mixture. In contrast, after being exposed to visible light for 20 minutes, the modified mesh was highly hydrophobic and could remove water from the same mixture. Fig. $2 \mathrm{~d}$ and e exhibit the removal results of the modified mesh under UV and visible light. Four different oils including $n$-hexane, toluene, gasoline and diesel were utilized to measure the removal property of the mesh. The oil residual rates for the four oils were lower than $0.1 \%$ under UV light, and the corresponding fluxes were higher than $4 \times 10^{5} \mathrm{~L} \mathrm{~m}^{-2} \mathrm{~h}^{-1}$, indicating the satisfactory oil removal property of the mesh under UV light. In comparison, the water residual rates were also lower than $0.1 \%$ under visible light, and the corresponding fluxes were about $1 \times 10^{5} \mathrm{~L} \mathrm{~m}^{-2} \mathrm{~h}^{-1}$, which were lower than for oil removal but still an acceptable result. Besides, in order to investigate the relationship between transformation variation and oil/water removal property, the meshes fabricated under different conditions were utilized to remove water from oil/ water mixtures (Fig. S17 †). The removal efficiencies of the meshes were $79.3 \%, 99.9 \%, 81.2 \%$, and $99.7 \%$, indicating that increasing the surface roughness could increase the transformation variation, and then improve the oil/water removal property of the mesh.

The as prepared mesh also showed satisfactory reusability and chemical/mechanical stability. Fig. 3a and the inset show the SEM images of the modified copper mesh after 20 successive removal processes. Almost no Ag pine needles had fallen off and the overall appearance remained unchanged. As shown in Fig. $3 \mathrm{~b}$, the oil/water removal efficiency remained higher than $99.80 \%$ after 10 times of oil removal and 10 times of water removal. Fig. S18† shows the XRD pattern of the modified copper mesh after 20 successive uses; no signal peak change was observed in the pattern. Fig. 3c illustrates the chemical stability of the modified mesh. The mesh was cut into strips and immersed in solutions of different $\mathrm{pH}$ values for $2 \mathrm{~h}$. The solutions were dyed using methyl orange to visually show their $\mathrm{pH}$ values. Then the strips were taken out and their WCAs under UV/Vis were measured. It was found that the mesh remained highly hydrophilic under UV light and highly hydrophobic
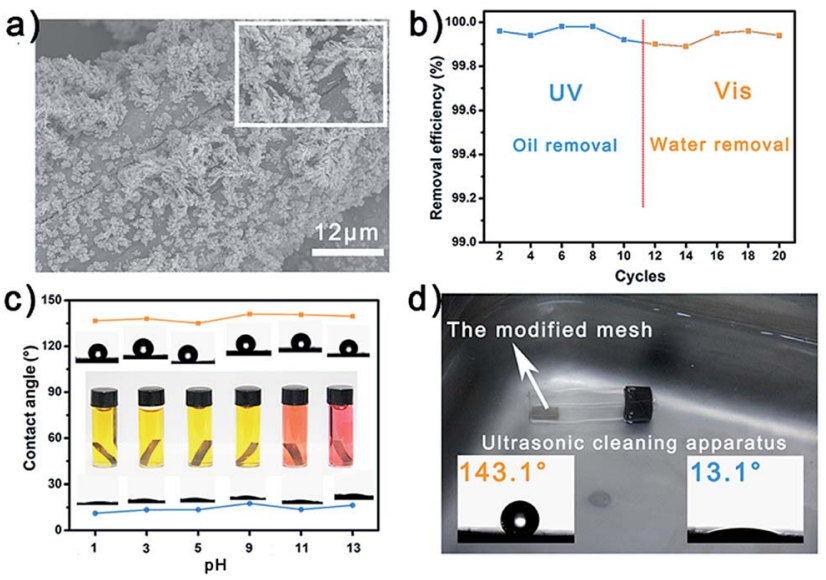

Fig. 3 The reusability and physical/chemical stability of the modified copper mesh: (a) SEM image of the modified copper mesh after 20 successive uses; (b) removal efficiency of 10 successive oil removal and 10 successive water removal cycles; (c) water contact angle of the modified copper mesh after 2 hours acid/alkaline solution treatment; (d) water contact angle of the modified copper mesh after 2 hours of ultrasonic treatment.

under visible light after acid and alkali treatment. The mechanical stability of the mesh was measured by treating the mesh with ultrasound for $2 \mathrm{~h}$ and measuring the WCAs. And the wetting behavior of the modified mesh remained unchanged (Fig. 3d). The salt resistance property of the mesh was also investigated. As shown in Fig. S19, $\dagger$ after being immersed in saturated $\mathrm{NaCl}$ solution for 2 hours, the mesh still remained highly hydrophobic under visible light and highly hydrophilic under UV light. In order to further demonstrate the durability of the mesh, the oil/water removal property of the mesh treated by different approaches (acid/alkaline treatment, ultrasonic treatment and salt treatment) were investigated, and the corresponding results are exhibited in Fig S20. $\dagger$ The water removal efficiency of the mesh decreased a little after the mesh was treated with strong acid/alkali, but the efficiencies were still acceptable. And for all of the meshes, the oil removal efficiency remained nearly unchanged. In this way, we could confirm the satisfactory selective oil/water removal property of the modified copper mesh.

\section{Modification of other conductive meshes}

According to the section on the photo-responsive wettability transformation, the wettability transformation property owed to the alternate exposure of the hydrophilic group (Ag) and hydrophobic group (amino-azo). The ability to selectively remove oil or water was based on the amplification effect on the wetting behavior, i.e., the utilization of small molecule AABN and the fractal Ag pine needles. Therefore, the key qualities for realizing the target function were the successful modification of amino-azo@Ag and the well-controlled surface structure. To validate this result, other commercial substrates were utilized in this strategy, including 400 mesh number stainless steel wire (400 SSM) and 1000 mesh number stainless steel wire (1000 SSM; they were different meshes with different constituents and 


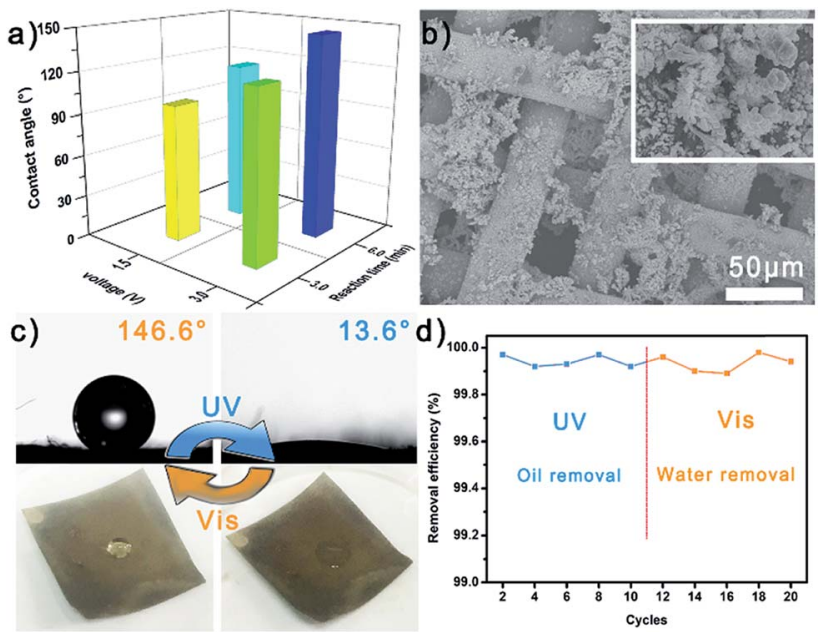

Fig. 4 Modification of 400 SSM: (a) the initial contact angles of modified 400 SSM fabricated under different conditions; (b) different magnifications of SEM images of modified $400 \mathrm{SSM}$; (c) photo response property of the modified 400 SSM; (d) removal efficiency of 10 successive oil removal and 10 successive water removal cycles using the modified 400 SSM.

structures). The pore sizes of the commercial conductive meshes are exhibited in Fig. S21. $\dagger$ Fig. 4 and S22 $\uparrow$ show the modification results of $400 \mathrm{SSM}$. The WCAs under different Ag fabrication conditions including $1.5 \mathrm{~V} / 3 \mathrm{~min}, 1.5 \mathrm{~V} / 6 \mathrm{~min}, 3 \mathrm{~V} /$ $3 \mathrm{~min}$ and $3 \mathrm{~V} / 6 \mathrm{~min}$ indicated that $3 \mathrm{~V} / 6 \mathrm{~min}$ was the optimum condition (Fig. 4a). Fig. 4b exhibits the SEM images of modified 400 SSM fabricated under optimum conditions, showing that the mesh surface was quite rough with dense $\mathrm{Ag}$ pine needles covered the mesh. The XRD patterns of the modified 400 SSM in different reaction stages are exhibited in Fig. S22. $\dagger$ The signal peak of Ag was firstly enhanced with the Ag pine needles being coated onto the mesh, then decreased with the AABN covering the Ag pine needles. The reversible photo-responsive property of the modified 400 SSM is shown in Fig. $4 \mathrm{c}$ and d. The modified 400 SSM was highly hydrophobic after exposure to visible light while highly hydrophilic after exposure to UV light. And the oil/ water removal efficiency remained higher than $99.80 \%$ after 10 cycles of oil removal and 10 cycles of water removal. Fig. S23 and $\mathrm{S} 24 \dagger$ show the corresponding results of modification on the 1000 SSM, which could also selectively remove oil or water. As a result, we could learn the universality of the facile approach, which made the approach easy-to-use.

\section{Conclusions}

In summary, we modified nano-Ag pine needles and AABN onto PDA pre-treated meshes, realizing a large-extent photoresponsive wettability transformation. The modified meshes were highly hydrophobic (WCA $\approx 150.0^{\circ}$ ) with water removal properties under visible light, and were highly hydrophilic $\left(\right.$ WCA $\approx 10.0^{\circ}$ ) with oil removal properties under $365 \mathrm{~nm} \mathrm{UV}$ light. The wettability transformation was reversible under alternate irradiation with UV and visible light. AABN with low steric hindrance and fractal $\mathrm{Ag}$ with high roughness were utilized to amplify the transformation extent. Besides, the modified meshes exhibited satisfactory removal efficiency, reusability and physical/chemical stability. Compared to previous works about photo-responsive azo compounds, this work showed a larger transformation extent in wetting behavior and could be applied to reversible removal between oil and water from oil/water mixtures.

\section{Experimental section}

\section{Materials}

The commercial copper meshes, 400 stainless steel meshes and 1000 stainless steel meshes (Sangon Biotech Co., Ltd., Shanghai, China) were used as purchased. Ferric trichloride $\left(\mathrm{FeCl}_{3} \cdot 6 \mathrm{H}_{2} \mathrm{O}\right.$, reagent pure, Beijing Chemical Co., Ltd., Beijing, China) and hydrochloric acid ( $\mathrm{HCl}$, Guaranteed reagent, Beijing Chemical Co., Ltd., Beijing, China) were purchased from Sinopharm Chemical Reagents. Silver nitrate $\left(\mathrm{Ag}_{3} \mathrm{NO}_{3}, 99 \%\right)$ was purchased from Xiya Chemical Co., Ltd. In addition, aminoazobenzene $\left(\mathrm{C}_{12} \mathrm{H}_{11} \mathrm{~N}_{3}, 98 \%\right)$ was purchased from Meryer Co., Ltd. Dopamine-hydrochloride (Sangon Biotech Co. Ltd, Shanghai, China) was used as purchased. And tris-(hydroxymethyl)aminomethane (Tris) was of analytical grade from Sinopharm Chemical Reagents. No further purification process was employed for all other reagents.

\section{Facile modification of various conductive meshes}

The conductive meshes (copper meshes, 400 SSM and 1000 SSM) were cut into squares with $4 \mathrm{~cm}$ lengths and then were ultrasonically washed in deionized water and acetone. An activation process was utilized to thoroughly clean the SSM. Then, polydopamine coated meshes were obtained by pretreating the meshes with dopamine-hydrochloride and tris-(hydroxymethyl) aminomethane. An electrolytic deposition process was conducted to modify Ag pine needles onto the PDA pretreated meshes, in which the meshes acted as the cathode while a clean copper sheet acted as the anode, and a $0.005 \mathrm{M} \mathrm{Ag}_{3} \mathrm{NO}_{3}$ solution was utilized as the electrolyte. The resultant nano-Ag-coated meshes were immersed into a $2 \mathrm{mM}$ aminoazobenzene solution under $60{ }^{\circ} \mathrm{C}$ for $24 \mathrm{~h}$ to modify with a layer of aminoazobenzene. Finally, the modified meshes were removed from the solution, washed with deionized water several times and dried.

\section{Photo-responsive oil/water removal}

A $365 \mathrm{~nm}$ UV flashlight and a visible light flashlight were utilized as the UV light source and visible light source, respectively. The modified meshes were exposed to UV light for 20 minutes to transform into a highly hydrophilic one, and were exposed to visible light for 20 minutes to transform into a highly hydrophobic one. In the oil/water removal experiments, two Teflon fixtures attached to a glass tube (with a diameter of 30 $\mathrm{mm}$ ) were utilized to fix the meshes. An oil/water mixture was poured onto the meshes. When the meshes were highly hydrophobic, oil smoothly passed through the mesh pores, 
while the water was isolated on the fixtures. In contrast, when the meshes were highly hydrophilic, water could pass through the mesh pores while oil was isolated. The separation efficiency was calculated by the equation $R=\left(1-C_{\text {filtrate }} / C_{\text {original }}\right) \times$ $100 \%$, in which $C_{\text {filtrate }}$ means the oil/water content in the water/ oil filtrate, and $C_{\text {original }}$ means the oil/water content in the original oil/water mixture.

\section{Characterization}

Scanning electron microscopy (SEM) images of the as-prepared meshes were obtained using field emission scanning electron microscopy (FESEM) (SU-8010, Hitachi Limited, Japan). X-ray diffraction (XRD) patterns were obtained on a polycrystalline $\mathrm{X}$-ray diffractometer with a $\mathrm{Cu} \mathrm{K} \alpha$ radiation source (Bruker D8 Advance, Bruker-AXS, Germany). X-ray photoelectron spectroscopy (XPS) data were obtained with a Thermo ESCALAB 250Xi spectrometer using an $\mathrm{Al} \mathrm{K \alpha}$ X-ray source $(1486.6 \mathrm{eV})$. The water and oil contact angles were measured using an OCA15 machine (Data-Physics, Germany) at ambient temperature. The oil content in the water filtrate was measured with an infrared (IR) spectrometer oil content analyzer (Oil480, Beijing Chinainvent Instrument Tech. Co., Ltd., China). The water content in the oil filtrate was measured using a Karl Fischer Titrator (Cou-Lo Aquamax KF Moisture Meter, UK). Optical microscopy images were acquired on a polarizing microscope (Nikon ECLIPSE LV100POL, Japan). The aminoazobenzene concentration in different solutions was tested with a PerkinElmer Lambda-750 UV spectrometer (United Kingdom). Energy-dispersive X-ray (EDX) images were measured using EDX analysis (Horiba, Ltd., Japan).

\section{Conflicts of interest}

There are no conflicts to declare.

\section{Acknowledgements}

The authors are grateful for financial support from the National Natural Science Foundation (51173099).

\section{Notes and references}

1 L. Feng, Z. Zhang, Z. Mai, Y. Ma, B. Liu, L. Jiang and D. Zhu, Angew. Chem., Int. Ed., 2004, 43, 2012-2014.

2 Q. Wen, J. Di, L. Jiang, J. Yu and R. Xu, Chem. Sci., 2013, 4, 591-595.

3 Z. Shi, W. Zhang, F. Zhang, X. Liu, D. Wang, J. Jin and L. Jiang, Adv. Mater., 2013, 25, 2422-2427.

4 M. Tao, L. Xue, F. Liu and L. Jiang, Adv. Mater., 2014, 26, 2943-2948.

5 H. Zhu, D. Chen, N. Li, Q. Xu, H. Li, J. He and J. Lu, Appl. Catal., B, 2017, 200, 594-600.

6 S. Wang, X. Feng, J. Yao and L. Jiang, Angew. Chem., Int. Ed., 2006, 45, 1264-1267.

7 S. He, H. Wang, C. Zhang, S. Zhang, Y. Yu, Y. Lee and T. Li, Chem. Sci., 2019, 10, 1816-1822.
8 Q. Wen, J. Di, Y. Zhao, Y. Wang and L. Jiang, Chem. Sci., 2013, 4, 4378-4382.

9 F. Li, Z. Wang, S. Huang, Y. Pan and X. Zhao, Adv. Funct. Mater., 2018, 28, 1801994.

10 C. Wu, X. Huang, X. Wu, R. Qian and P. Jiang, Adv. Mater., 2013, 25, 5658-5662.

11 W. Zhang, N. Liu, Y. Cao, Y. Chen, L. Xu, X. Lin and L. Feng, Adv. Mater., 2015, 27, 7349-7355.

12 W. Zhang, N. Liu, Y. Cao, X. Lin, Y. Liu and L. Feng, Adv. Mater. Interfaces, 2017, 4, 1700029.

13 D. Zang, R. Zhu, W. Zhang, X. Yu, L. Lin, X. Guo, M. Liu and L. Jiang, Adv. Funct. Mater., 2017, 27, 1605446.

14 X. Li, Q. Zhang, W. Zhang, R. Qu, Y. Wei and L. Feng, Adv. Mater. Interfaces, 2018, 5, 1801179.

15 X. Lin, F. Lu, Y. Chen, N. Liu, Y. Cao, L. Xu, W. Zhang and L. Feng, Chem. Commun., 2015, 51, 16237-16240.

16 M. Cheng, Q. Liu, G. Ju, Y. Zhang, L. Jiang and F. Shi, Adv. Mater., 2014, 26, 306-310.

17 F. Mugele, A. Klingner, J. Buehrle, D. Steinhauser and S. Heiminghaus, J. Phys.: Condens. Matter, 2005, 17, S559S576.

18 W. Zhang, N. Liu, Q. Zhang, R. Qu, Y. Liu, X. Li, Y. Wei, L. Feng and L. Jiang, Angew. Chem., Int. Ed., 2018, 57, 5740-5745.

19 A. Nayak, H. Liu and G. Belfort, Angew. Chem., Int. Ed., 2006, 45, 4094-4098.

20 D. Kim, M. Jung, S. Cho, S. Kim, H. Kim, H. Lee, K. Oh and M. Moon, Sci. Rep., 2015, 5, 12908.

21 R. Qu, W. Zhang, X. Li, Y. Liu, T. Shih, Y. Wei and L. Feng, J. Mater. Chem. A, 2018, 6, 18003-18009.

22 O. Bushuyev, T. Corkery, C. Barrett and T. Friščić, Chem. Sci., 2014, 5, 3158-3164.

23 P. Mondal, G. Granucci, D. Rastadter, M. Persice and I. Burghardt, Chem. Sci., 2018, 9, 4671-4681.

24 C. Yang, C. Slavov, H. Wegner, J. Wachtveitl and A. Dreuw, Chem. Sci., 2018, 9, 8665-8672.

25 Y. Yan, H. Wang, B. Li, G. Hou, Z. Yin, L. Wu and V. Yam, Angew. Chem., Int. Ed., 2010, 49, 9233-9236.

26 T. Ishikawa and T. Noro, J. Chem. Phys., 2001, 115, 75037512.

27 H. Rao and E. Luddecke, J. Am. Chem. Soc., 1982, 104, 16161620.

28 G. Xie, P. Li, Z. Zhao, Z. Zhu, X. Kong, Z. Zhang, K. Xiao, L. Wen and L. Jiang, J. Am. Chem. Soc., 2018, 140, 4552-4559.

29 G. Petroffe, C. Wang, X. Sallenave, G. Sini, F. Goubard and S. Peralta, J. Mater. Chem. A, 2015, 3, 11533-11542.

30 G. Whyman, E. Bormashenko and T. Stein, Chem. Phys. Lett., 2008, 450, 355-359.

31 G. Wolansky and A. Marmur, Colloids Surf., A, 1999, 156, 381-388.

32 K. Ichimura, S. Oh and M. Nakagewa, Science, 2000, 288, 1624-1626.

33 H. Lim, J. Han, D. Kwak, M. Jin and K. Cho, J. Am. Chem. Soc., 2006, 128, 14458-14459.

34 H. Zhang, L. Bre, T. Zhao, Y. Zheng, B. Newland and W. Wang, Biomaterials, 2014, 35, 711-719. 
35 R. Qu, W. Zhang, N. Liu, Q. Zhang, Y. Liu, X. Li, Y. Wei and L. Feng, ACS Sustainable Chem. Eng., 2018, 6, 8019-8028.

36 N. Liu, R. Qu, Y. Chen, Y. Cao, W. Zhang, X. Lin, Y. Wei, L. Feng and L. Jiang, Nanoscale, 2016, 8, 18558-18564.

37 B. Coyle, M. McCann, K. Kavanagh, M. Devereux, V. Mckee, N. Kayal, D. Egan, C. Deegan and G. Finn, J. Inorg. Biochem., 2004, 98, 1361-1366.

38 Q. Liu, Y. Wang, W. Li and L. Wu, Langmuir, 2007, 23, 82178223.
39 R. Jansen and H. Bekkum, Carbon, 1995, 33, 1021-1027.

40 T. Okpalugo, P. Papakonstantinou, H. Murphy, J. McLaughlin and N. Brown, Carbon, 2005, 43, 153-161.

41 I. Salido, D. Lim and Y. Kim, Surf. Sci., 2005, 588, 6-18.

42 S. Maldonado, S. Morin and K. Stevenson, Carbon, 2006, 44, 1429-1437.

43 D. Philip, Spectrochim. Acta, Part A, 2009, 73, 374-381. 